\title{
Planetesimal and protoplanet dynamics in a turbulent protoplanetary disk
}

\author{
Chao-Chin Yang ${ }^{1,2} \dagger$, Mordecai-Mark Mac Low ${ }^{2}$ and Kristen Menou ${ }^{3}$ \\ ${ }^{1}$ Department of Astronomy, University of Illinois, \\ 1002 West Green Street, Urbana, IL 61801, U.S.A \\ ${ }^{2}$ Department of Astrophysics, American Museum of Natural History, \\ Central Park West at 79th Street, New York, NY 10024, U.S.A \\ ${ }^{3}$ Department of Astronomy, Columbia University, \\ 550 West 120th Street, New York, NY 10027, U.S.A
}

\begin{abstract}
Due to the gravitational influence of density fluctuations driven by magnetorotational instability in the gas disk, planetesimals and protoplanets undergo diffusive radial migration as well as changes of other orbital properties. The magnitude of the effect on particle orbits has important consequences for planet formation scenarios. We use the local-shearingbox approximation to simulate an ideal, isothermal, magnetized gas disk with vertical density stratification and simultaneously evolve numerous massless particles moving under the gravity of the gas and the host star. Although the results converge with resolution for fixed box dimensions, we find there exists no convergence of the response of the particles to the gravity of the gas against the horizontal box size, up to 16 disk scale heights. This lack of convergence indicate that caution should be exercised when interpreting local-shearing-box models involving gravitational physics of magneto-rotational turbulence.
\end{abstract}

Keywords. accretion, accretion disks, instabilities, methods: numerical, MHD, planetary systems: formation, planetary systems: protoplanetary disks, turbulence

\section{Introduction}

It is generally believed that turbulent accretion must operate in protoplanetary gas disks. One of the most promising mechanisms to drive the turbulence is the magnetorotational instability (MRI; see, e.g., Balbus \& Hawley 1998). The resulting density fluctuations are significant enough that the gravitational influence of the turbulent gas makes the embedded planetesimals or protoplanets undergo diffusive radial migration as well as changes of other orbital properties. The magnitude of this effect on particle orbits has important consequences for planet formation scenarios.

To self-consistently measure the influence of the turbulent density fluctuations on particle orbital dynamics, numerical simulations capturing both large-scale and small-scale coherent structures as well as statistical techniques to quantify stochastic particle orbital evolution are required. We adopt the local-shearing-box approximation, which has highresolving power, can be integrated for long physical timescale, and can simultaneously involve a significant number of particles.

We presented in Yang et al. (2009) our simulations of massless particles moving in a local, unstratified, isothermal, ideal, magnetized disk. We found that the response of the particles to the gravity of turbulent gas subject to the MRI is systematically lower than what was previously reported in global disk models. We also found in the same study

$\dagger$ Present address: Department of Astronomy and Astrophysics, University of California, Santa Cruz, 1156 High Street, Santa Cruz, CA 95064, U.S.A.; email: ccyang@ucolick.org 

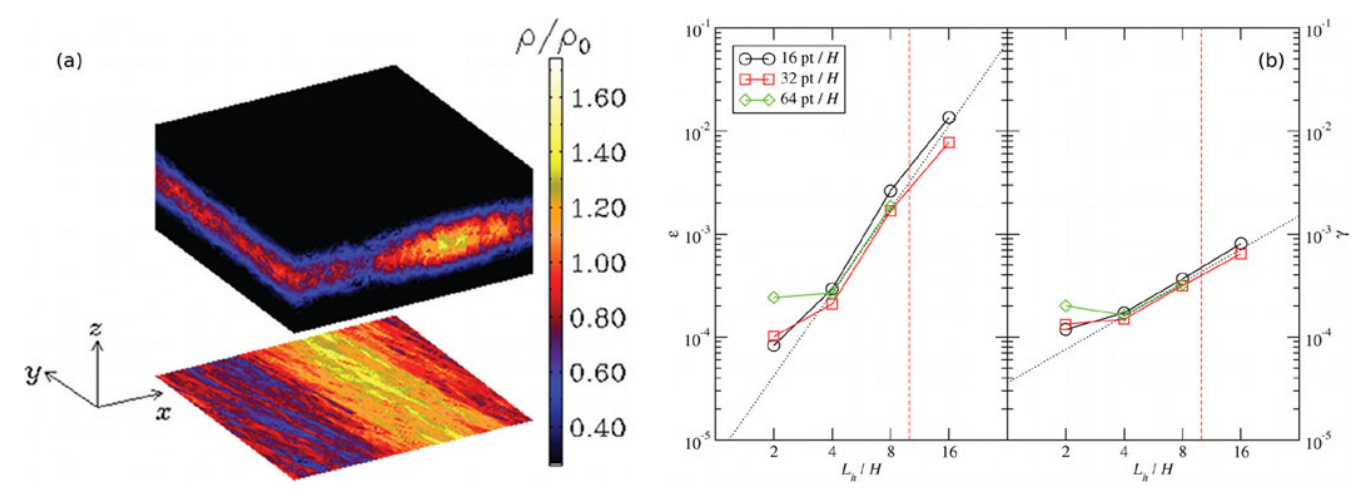

Figure 1. (a) Snapshot of the gas density of a local region in a turbulent protoplanetary disk driven by the magneto-rotational instability. The $x, y$, and $z$ axes indicate radial, azimuthal, and vertical directions, respectively. The bottom plane shows the slice through the disk mid-plane. The dominance of large-scale structures can be seen; this type of structures are typical in our simulations and have long correlation time compared with orbital period. (b) Dimensionless constants $\epsilon$ and $\gamma$ as a function of horizontal box size $L_{h}$. The constant $\epsilon$ represents the strength of radial diffusive migration driven by turbulence and was defined by Johnson et al. (2006), while $\gamma$ is related with the strength of eccentricity excitation due to turbulence and was defined by Ida et al. (2008). The dotted lines are power-law fits to data points with $L_{h} \geqslant 4 H$. The vertical dashed lines indicate the box size of choice for the case of a disk aspect ratio of $H / R=0.1$.

that the effect significantly depends on the horizontal box size. In this work, we further consider disks with vertical density stratification by including linearized vertical gravity from the central star.

\section{Results and Discussions}

We find that no matter what the horizontal box size is, the largest-scale structure in either radial or azimuthal direction dominates the density fluctuations in the gas, up to the largest box we have investigated (Fig. 1a; also see Johansen et al. 2009). These largescale density structures significantly affect the stochastic evolution of particle orbital radius and eccentricity (Fig. 1b). Both the magnitude and the correlation time of the resulting stochastic torques increase with horizontal box size $L_{h}$. Consequently, the larger the shearing box, the stronger the response of the particles to the gravity of the turbulent gas. We see no convergence with $L_{h}$ up to $16 H$, where $H$ is the vertical disk scale height.

This lack of convergence in particle dynamics in shearing boxes poses major difficulty in quantifying the influence of the magneto-rotational turbulence on particle orbits. Using heuristic arguments, we suggest that $L_{h} \sim R$, where $R$ is the distance of the box center to the host star, might be a natural scale of choice for a local model to approach reality. Comparison to recent global disk models conducted by Nelson \& Gressel (2010) suggests this criterion offers consistency between local and global disk models.

\section{References}

Balbus, S. A. \& Hawley, J. F. 1998, Reviews of Modern Physics, 70, 1

Ida, S., Guillot, T., \& Morbidelli, A. 2008, ApJ, 686, 1292

Johansen, A., Youdin, A., \& Klahr, H. 2009, ApJ, 697, 1269

Johnson, E. T., Goodman, J., \& Menou, K. 2006, ApJ, 647, 1413

Nelson, R. P. \& Gressel, O. 2010, MNRAS, 409, 639

Yang, C.-C., Mac Low, M.-M., \& Menou, K. 2009, ApJ, 707, 1233 